\title{
Zero's of Exponential Sums and Best Diophantine Approximations
}

\author{
By \\ GEORG LOHÖFER*, DIETER MAYER'
}

\begin{abstract}
We show that the location of the m'th zero of the exponential sum $g(z ; \beta)=e^{-z}+e^{-\beta z}-2$, $0 \leq \beta \leq 1$ depends monotonically on the Diophantine distance $\Delta_{m}=\min _{b \in Z}|m \beta-p|$ of the parameter $\beta$. The approach of the zero's of the above function to the imaginary axis is determined therefore by the best Diophantine approximation denominators $q_{n}$ of $\beta$.
\end{abstract}

\section{§1. Introduction and Results}

In several papers [1-4] treating the problems of analytic extensions of zeta functions respectively the closely related question of the decay of correlation functions for certain flows [13] exponential sums of the form $g(z ; \beta)=e^{-z}+e^{-\beta z}-2$ and especially the location of their zeros in the complex $z$-plane play an important role. Consider namely two observables $A$ and $B$ of such a flow $\phi_{t}: M \rightarrow M$ and their time correlation function

$$
\begin{array}{r}
C(t)=\lim _{T \rightarrow \infty} 1 / T \int_{0}^{T} A\left(\phi_{t+t^{\prime}} x\right) B\left(\phi_{t^{\prime}} x\right) d t^{\prime}-\lim _{T \rightarrow \infty} 1 / T \int_{0}^{T} A\left(\phi_{t^{\prime}} x\right) d t^{\prime} \\
\circ \lim _{T \rightarrow \infty} 1 / T \int_{0}^{T} B\left(\phi_{t^{\prime}} x\right) d t^{\prime} .
\end{array}
$$

For flows which are chaotic enough one expects their long time behaviour to be described by some asymptotic measure $\mu[1-4]$, [13] such that the above function can be written as

$$
C(t)=\int_{M} d \mu A\left(\phi_{t} x\right) B(x)-\int_{M} d \mu A(x) \int_{M} d \mu B(x)
$$

Communicated by H. Araki, July 26, 1985.

* Institut für Theoretische Physik, E;RWTH-Aachen, 51 Aachen, Fed. Rep. Germany, Supported by DFG grant Ma 633/4-3.

i Heisenberg Fellow. 
Its long time behaviour for $t \rightarrow \infty$ is closely related to the pole structure of its Laplace transform

$$
\hat{C}(z)=\int_{0}^{\infty} \exp -z t \quad G(t) d t
$$

In the cases of mixing Axiom-A Systems considered in [1-4] and a semiflow constructed as the suspension of a dissipative time discrete model [15] in [13], this Fourier-Laplace transform has a very simple form:

$$
\hat{C}(z)=\hat{A}(z) /\left(2-e^{-z}-e^{-\beta z}\right)+\hat{f}(z)
$$

where $\hat{f}$ and $\hat{A}$ are holomorphic functions and $\beta$ some positive, irrational parameter. The long time behaviour of $C(t)$ can be simply related to the pole structure of the function $\hat{C}(z)$ that means the zeros of the exponential sum $g(z ; \beta)$ :

$$
C(t) \underset{t \rightarrow \infty}{\sim} \sum_{m} \hat{A}_{m}\left(z_{m}\right) \exp z_{m} t
$$

where the sum is over all zero's of $g(z ; \beta)$ different from $z=0$. Their location therefore determines the exact decrease of $C(t)$. It is desirable to know the location of these zeros as precisely as possible if one wants to know the exact behaviour of $C(t)$ for large $t$.

Several facts about the zero's of exponential sums are known for quite a long time. These functions are special cases of analytic almost periodic functions of $\mathrm{H}$. Bohr [5] studied in great detail by Jessen and Tornehave [6]. Their results and also those earlier ones by Tamarkin [7], Polya [8], Ritt [9] and others (see the literature in [6]) deal mostly with global problems like the distribution of zero's in strips and the existence of densities. They also found that there exist lines such that all the zero's lie to its left or to its right, on which the function comes arbitrarily near to, or takes, the value zero. In our special case of the function $g(z ; \beta)$ one of those lines is the imaginary axis. For $\beta$ rational the function $g(z ; \beta)$ is periodic in the $y$-variable and infinitely many zero's lie on the imaginary axis. The correlation function therefore does not decay and is oscillating in time instead. For $\beta$ irrational besides $z=0$ the function $g(z ; \beta)$ does not vanish for $x=0$ but it takes values arbitrary near to zero; with other words, its zero's approach this axis arbitrarily close. From this fact both Ruelle and Pollicott concluded that $C(t)$ does not dacay 
exponentially fast for arbitrary mixing Axiom $A$ flows. In [2] $\mathrm{M}$. Pollicott constructed flows which decay slower than any predetermined rate. This happens for flows where the parameter $\beta$ can be approximated very well by rationals. Depending on the closeness of this rational to $\beta$ the correlation functions corresponding to the two values stay close for a long period.

In the present paper we study the location of the individual zero's of the function $g(z ; \beta)$, in contrast to the global aspects considered up to now in the literature primarily. Our results are restricted to the special case of exponential sums of the form $g(z ; \beta)$. It would be interesting to find out how far they can be extended to more general exponential sums or even to general analytic almost periodic functions.

Our main result is the following

Theorem I. For every $0 \leq \beta<1$ there exist bounded periodic real analytic functions $\xi_{\beta}: \boldsymbol{R} \rightarrow \boldsymbol{R}, \eta_{\beta}: \boldsymbol{R} \rightarrow \boldsymbol{R}$ with period 1 with the properties:

a) $\xi_{\beta}(\Delta)=\xi_{\beta}(-\Delta), \eta_{\beta}(\Delta)=-\eta_{\beta}(-\Delta)$ for all $\Delta \in \mathbb{R}$

$\xi_{\beta}(0)=0, \xi_{\beta}(1 / 2)=x_{0}(\beta), \eta_{\beta}(0)=0, \eta_{\beta}(1 / 2)=0$

(where $x_{0}(\beta)$ is the real solution of $e^{-x}-e^{-\beta x}-2=0$ )

$x_{0}(\beta) \leq \xi_{\beta}(\Delta) \leq 0, \quad\left|\eta_{\beta}(\Delta)\right| \leq \pi / 2$ for all $\Delta \in \mathbb{R}$

b) $\frac{d}{d \Delta} \xi_{\beta}(\Delta)=\xi_{\beta}^{\prime}(\Delta) \leq 0$ for all $0 \leq \Delta \bmod 1 \leq 1 / 2$

$\eta_{\beta}^{\prime}(\Delta) \leq 0$ for all $0 \leq \Delta \bmod 1 \leq \tilde{\Delta}$ for some $\tilde{\Delta}$ with $0<\tilde{\Delta}<1 / 2$.

$\xi_{\beta}^{\prime}(0)=\xi_{\beta}^{\prime}(1 / 2)=0, \quad \eta_{\beta}^{\prime}(0)=-2 \pi /(\beta+1)$,

$\eta_{\beta}^{\prime}(1 / 2)=2 \pi e^{-\beta x_{0}(\beta)} /\left(e^{-x_{0}(\beta)}-\beta e^{-\beta x_{0}(\beta)}\right) ; \xi_{\beta}^{\prime \prime}(0)=-8 \pi^{2} /(\beta+1)^{3}$,

such that any zero $z_{m}$ of $g(z ; \beta)$ can be written as

$$
z_{m}=\xi_{\beta}\left(\Delta_{m}\right) \pm i \eta_{\beta}\left(\Delta_{m}\right)+2 \pi i m, m \in \mathbb{Z}
$$

where the "+" sign corresponds to those $m$ such that $0 \leq m \beta-[m \beta] \leq \frac{1}{2}$ and the "-" sign to those with $1>m \beta-[m \beta] \geq \frac{1}{2} . \Delta_{m}$ denotes the Diophantine distance of $\beta$ :

$$
\Delta_{m}=\min _{p \in \boldsymbol{Z}}|m \beta-p| .
$$

Remarks. (1) For $\beta=1$ the zero's of $g(z ; 1)$ are simply given by the formula $z_{m}=i 2 \pi m$ and the Theorem becomes trivial. 
(2) For $\beta=p / q$ we know that $\Delta_{m}$ is periodic in $m$ with period $q: \Delta_{m}$ $=\Delta_{m+q^{*}}$ Because $\Delta_{0}=0$ for all $n: \Delta_{n q}=0$ and all the zero's are periodically located along the positive and negative imaginary axis with every $q$-th of them on this axis.

(3) Inside every rectangle

$$
\mathfrak{R}_{m}^{\varepsilon}=\left\{x+i y:-x_{0}-\varepsilon \leq x \leq \varepsilon,\left(2 m-\frac{1}{2}\right) \pi \leq y \leq\left(2 m+\frac{1}{2}\right) \pi\right\}, \varepsilon>0
$$

there is exactly one zero of $g(z ; \beta)$.

(4) The distance of the point $z_{m}$ from the imaginary axis given by $\left|x_{m}\right|$ is a strictly monotone function of the Diophantine distance $\Delta_{m}:\left|x_{m^{\prime}}\right|<\left|x_{m}\right|$ if and only if $\Delta_{m}<\Delta_{m^{\prime}}$. From this it follows that the approach of the zero's to the imaginary axis is determined exactly by those $z_{m}$ whose $m$ is a best Diophantine approximation denominator for $\beta$. These numbers are inductively defined by $q_{0}=1$ and the property that $\Delta_{m}>\Delta_{q_{n}}$ for all $q_{n-1} \leq m<q_{n}$. It is known that these $q_{n}$ are determined by the $\mathrm{n}$-th convergents of the continued fraction expansion of $\beta[10]$. For $n \rightarrow \infty$ we find

$$
x_{q_{n}} \sim-8 \pi^{2} /(\beta+1)^{3} \Delta_{q_{n}}^{2}+0\left(\Delta_{q_{n}}^{4}\right) \text {. }
$$

(5) The distance of the point $z_{m}$ from the line $y=2 m \pi$ given by $\left|y_{m}-2 m \pi\right|$ is also a stricly monotone function of $\Delta_{m}$ at least for $\Delta_{m}$ small enough: $\left|\eta_{\beta}\left(\Delta_{m}\right)\right|<\left|\eta_{\beta}\left(\Delta_{m^{\prime}}\right)\right|$ if and only if $\Delta_{m}<\Delta_{m^{\prime}}$ for all $\Delta_{m}, \Delta_{m^{\prime}} \leq \tilde{\Delta}_{\text {. }}$ Asymptotically we get for large $n: \eta_{\beta}\left(\Delta_{q_{n}}\right)=$ $\frac{2 \pi}{\beta+1} \Delta_{q_{n}}+0\left(\Delta_{q_{n}}^{3}\right)$. For reasons of simplicity we omit the index $\beta$ in the functions $\xi_{\beta}, \eta_{\beta}$ from now on.

(4) and (5) together tell us that as the zero's $z_{m}$ approach the imaginary axis they also approach the centre of the corresponding rectangle $\mathscr{R}_{m}^{\varepsilon}$ with respect to the $y$ coordinate.

\section{§2. Proof of the Main Theorem}

Let $\Delta$ be any real and denote by $g(z ; \beta ; \Delta)$ the exponential sum

$$
g(z ; \beta ; \Delta)=e^{-z}+e^{-\beta z-2 \pi i \Delta}-2 \text { 。 }
$$


For $\Delta=0$ appearently $g(z ; \beta ; 0)=g(z ; \beta)$. If $\zeta(\Delta)=\xi(\Delta)+i \eta(\Delta)$ denotes a zero of $g(z ; \beta ; \Delta)$ then $\zeta^{*}(\Delta)$ is a zero of the function $g(z ; \beta ;-\Delta)$ and vice versa. Choose next a number $L_{1}>0$ such that $e^{2 L_{1}}-2 e^{L_{1}}>$ $e^{2 \beta L_{1}}$ which for $0 \leq \beta<1$ is always possible. If $x_{0}(\beta)$ denotes the real solution of the equation $e^{-x}-e^{-\beta x}=2$ let $L$ be a positive number $L>\max \left\{L_{1},\left|x_{0}(\beta)\right|\right\}$. For $\varepsilon>0$ denote by $\mathscr{R}_{\varepsilon, L}$ the closed rectangle

$$
\mathscr{R}_{\varepsilon, L}=\{\zeta=\xi+i \eta:-L \leq \xi \leq \varepsilon,-\pi \leq \eta \leq \pi\} \text { 。 }
$$

Then we can show

Theorem $\mathbb{H}$. For every $\Delta \in \mathbb{R}$ the function $g(z ; \beta ; \Delta)$ has exactly one zero $\zeta(\Delta)=\xi(\Delta)+i \eta(\Delta)$ in the strip $-\frac{\pi}{2} \leq \eta \leq \frac{\pi}{2}$ which even lies inside the rectangle $\mathscr{R}_{\varepsilon, L}$ for every $\varepsilon>0$. The mapping $\Delta \rightarrow \zeta(\Delta)$ is a periodic real analytic mapping with period 1 and has the following properties

a) $\zeta(-\Delta)=\zeta(\Delta)$

b) $\zeta(0)=0, \zeta(1 / 2)=x_{0}(\beta)$

c) $\zeta^{\prime}(0)=-i 2 \pi /(\beta+1), \zeta^{\prime}(1 / 2)=2 \pi i e^{-\beta x_{0}(\beta)} /\left(e^{-x_{0}(\beta)}-\beta e^{-\beta x_{0}(\beta)}\right)$

d) $\frac{d \xi(\Delta)}{d \Delta} \leq 0$ for $0 \leq \Delta \bmod 1 \leq 1 / 2, \xi(\Delta)=0$ iff $\Delta=n$

$\frac{d \eta(\Delta)}{d \Delta} \leq 0$ for $0 \leq \Delta \bmod 1 \leq \tilde{\Delta}$ with some $0<\tilde{\Delta}<1 / 2$.

From this Theorem I follows immediately:

We can restrict ourselves to the zero's in the upper half plane $\mathbb{C}_{+}$: For $z_{m} \in \mathbb{C}_{+}$with $(2 m-1) \pi \leq y_{m} \leq(2 m+1) \pi, m \geq 0$ we can write $z_{m}$ $=\zeta_{m}+2 \pi i m$ where $\zeta_{m}$ lies in the strip $-\pi \leq \eta_{m} \leq \pi$. Therefore

$$
g\left(z_{m} ; \beta\right)=e^{-\zeta_{m}}+e^{-\beta \zeta_{m}-2 \pi i m \beta}-2 \text {. }
$$

The zero's of the function $g(z ; \beta)$ in the strip $(2 m-1) \pi \leq y \leq(2 m+1) \pi$ are therefore related to the zero's of the function $g(\zeta ; \beta ; m \beta)$ in the strip $-\pi \leq \eta \leq \pi$. Theorem II tells us that there exists exactly one zero $\zeta(m \beta)$ for the latter function in $\mathscr{R}_{0, L}$ which only for rational $\beta$ can lie on the imaginary axis. Because of the symmetry and periodicity of the function $\zeta(\Delta)$ we get, by using the fact that $m \beta-[m \beta]=\Delta_{m}$ if the left hand side is smaller than $\frac{1}{2}$, the result

$$
\zeta(m \beta)=\zeta\left(\Delta_{m}\right) \text { respectively, }
$$




$$
\zeta(m \beta)=\zeta\left(-\Delta_{m}\right) \text { for } m \beta-[m \beta]=1-\Delta_{m} \text {. }
$$

The zero's of $g(z ; \beta)$ can therefore be represented in the form claimed in Theorem I and have all the desired properties following from the properties of the function $\zeta(\Delta)$ in Theorem II.

The proof of Theorem II is divided into several Lemmatas.

Lemma 1. For every $\Delta \in \boldsymbol{R}$ there exists exactly one zero $\zeta(\Delta)$ of $g(z ; \beta ; \Delta)$ in the strip $-\pi \leq \eta \leq \pi$. More precisely $\zeta(\Delta) \in$ Int $\mathscr{R}_{\varepsilon, L}$ for all $\varepsilon>0$ and $\zeta(\Delta) \in$ Int $\mathscr{R}_{0, L}$ if $\Delta \neq n$.

Proof. Periodicity of $g(z ; \beta ; \Delta)$ in $\Delta$ allows us to restrict the values of $\Delta$ to the interval $-\frac{1}{2} \leq \Delta \leq \frac{1}{2}$. If $g(z)$ denotes the function $g(z)$ $=e^{-z}-2$ then its zeros can be written down immediately: $z_{m}=$ $-\ln 2+2 \pi i m, m \in \boldsymbol{Z}$. Only the zero $z_{0}=-\ln 2$ lies in the strip $-\pi \leq \eta \leq \pi$ and trivially $z_{0} \in$ Int $R_{0, L}$. We want to apply a Theorem of Poincaré and Bohl [11] respectively of Kronecker [12] to the functions $\mathrm{g}(z)$ and $g(z ; \beta ; \Delta)$ on the rectangle $\mathscr{R}_{\varepsilon, L}$ for some $\varepsilon>0$. To do this we have to show that for all $z \in \partial \mathscr{R}_{\varepsilon, L}$ the point $0 \in C$ does not belong to the straight line $\overline{[g(z), g(z ; \beta ; \Delta)]}$ connecting the two points in $\boldsymbol{C}$. For $y= \pm \pi,-L \leq x \leq \varepsilon$ we get for $g(z)$ :

$$
g(z)=-e^{-x}-2
$$

respectively for $g(z ; \beta ; \Delta)$ :

$$
g(z ; \beta ; \Delta)=-e^{-x}-2+e^{-\beta x}(\cos (\beta \pi+2 \pi \Delta) \mp i \sin (\beta \pi+2 \pi \Delta)) .
$$

Because both $\operatorname{Re} g(z)$ and $\operatorname{Re} g(z ; \beta ; \Delta)$ are obviously strictly negative $(\beta<1$ !) we have $0 \notin \overline{[g(z), g(z ; \beta ; \Delta)]}$. Consider next the boundary: $x=\varepsilon ;-\pi \leq y \leq \pi$. There

$$
g(z)=e^{-\varepsilon}(\cos y-i \sin y)-2
$$

respectively

$$
g(z ; \beta ; \Delta)=e^{-\varepsilon}(\cos y-i \sin y)-2+e^{-\beta \varepsilon}(\cos (\beta y+2 \pi \Delta)-i \sin (\beta y+2 \pi \Delta)) .
$$

Again both $\operatorname{Re} g(z)$ and $\operatorname{Re} g(z ; \beta ; \Delta)$ are strictly negative (remember $\varepsilon>0)$ and hence $0 \notin \overline{[g(z), g(z ; \beta ; \Delta)]}$. There remains to consider only the boundary $x=-L,-\pi \leq y \leq \pi$. Because $e^{2 L}-2 e^{L}>e^{2 \beta L}$ we get $|g(z)|>\left|e^{-\beta z-2 \pi i \Delta}\right|=e^{2 \beta L}$. The Theorem of Poincaré and Bohl shows then that the function $g(z ; \beta ; \Delta)$ has exactly as many zero's inside the 
rectangle $\mathscr{R}_{\varepsilon, L}$ for any $\varepsilon>0$ as the function $g(z)$. To see that there does not exist any further zero of $\mathrm{g}(z ; \beta ; \Delta)$ in the strip $-\pi \leq y \leq \pi$ we only have to repeat the above reasoning for the rectangle $\mathscr{R}_{\varepsilon, L}$ for bigger and bigger $\varepsilon$ and $L$. Let us next assume that $\zeta(\Delta)$ lies on the imaginary axis, that means $\xi(\Delta)=0$. This leads to the equation

$$
\cos \eta(\Delta)-i \sin \eta(\Delta)+\cos (\beta \eta(\Delta)+2 \pi \Delta)-i \sin (\beta \eta(\Delta)+2 \pi \Delta)-2=0
$$

and therefore to

$$
\cos \eta(\Delta)+\cos (\beta \eta(\Delta)+2 \pi \Delta)=2
$$

For $-\pi \leq y \leq \pi$ the only solution is $\eta(\Delta)=0$ and $\Delta=n$, For $\Delta \neq n$ hence $\xi(\Delta)<0$ because $\zeta(\Delta) \in \mathscr{R}_{0, L}$ for all $\Delta$. We can improve this result even more: the function $\eta(\Delta)$ is bounded in absolute value by $\frac{\pi}{2}$. That means for every $\Delta$ the zero of $g(z ; \beta ; \Delta)$ in $\mathscr{R}_{0, L}$ lies even in the substrip $-\pi / 2<y<\pi / 2$. For, assume that $|\eta(\Delta)| \geq \pi / 2$. Then the equation $g(z ; \beta ; \Delta)=0$ can be written as

$$
\begin{aligned}
& e^{-x} \cos y+e^{-\beta x} \cos (\beta y+2 \pi \Delta)=2 \\
& e^{-x} \sin y+e^{-\beta x} \sin (\beta y+2 \pi \Delta)=0
\end{aligned}
$$

and therefore

$$
\begin{aligned}
& \sin (\beta y+2 \pi \Delta)=-e^{(\beta-1) x} \sin y \\
& \cos (\beta y+2 \pi \Delta)=2 e^{\beta x}-e^{(\beta-1) x} \cos y .
\end{aligned}
$$

For $x=\xi(\Delta)$ and $y=\eta(\Delta)$ we get for $\pi \geq|\eta(\Delta)| \geq \frac{\pi}{2}$ : $\cos \eta(\Delta) \leq 0$ and therefore $\cos (\beta \eta(\Delta)+2 \pi \Delta)>-e^{(\beta-1) x} \cos y \geq 0$. Squaring both sides and adding the two lines leads to the result

$$
1>e^{(\beta-1) \xi(\Delta)} \text { contrary to the relation } \xi(\Delta) \leq 0 \text {. }
$$

Corollary. For $\beta$ irrational no zero of $g(z ; \beta)$ besides $z=0$ lies on the imaginary axis.

Proof. Follows from the fact that $\mathrm{m} \beta \neq n$ for all $\mathrm{m}, n \in \mathbb{Z} \backslash\{0\}$.

Lemma 2. The function $\Delta \rightarrow \zeta(\Delta)$ is real analytic, periodic of period 1 and fulfills the relation

$$
\zeta(-\Delta)=\zeta(\Delta) *
$$

Proof. The function $g(z ; \beta ; \Delta)$ is both holomorphic in $z$ and real 
analytic in $\Delta$. From Lemma 1 we know that $g(\zeta(\Delta) ; \beta ; \Delta)=0$ for all $\Delta \in \mathbb{R}$. We only have to make sure that $\left.\frac{\partial g(z ; \beta ; \Delta)}{\partial z}\right|_{z=\xi(\Delta)} \neq 0$ for all 4. But the left hand side is simply

$$
\frac{\partial g(z ; \beta ; \Delta)}{\partial z}=-e^{-z}-\beta e^{-\beta z-2 \pi i \Delta} .
$$

For $\operatorname{Re} z \leq 0$ obviously $\left|e^{-z}\right|>\beta\left|e^{-\beta z}\right|$ because $0 \leq \beta<1$ and therefore $\left.\frac{\partial g(z ; \beta ; \Delta)}{\partial z}\right|_{z=\zeta(\Delta)} \neq 0$ because $\operatorname{Re} \zeta(\Delta) \leq 0$. The Implicit Function Theorem for real analytic mappings then shows that $\zeta(\Delta)$ is real analytic in $\Delta$ and that furthermore

$$
\frac{d \zeta(\Delta)}{d \Delta}=-\left.\frac{\partial g(z ; \beta ; \Delta)}{\partial \Delta}\right|_{z=\zeta(\Delta)} /\left.\frac{\partial g(z ; \beta ; \Delta)}{\partial z}\right|_{z=\zeta(\Delta)} .
$$

Because of the uniqueness of $\zeta(\Delta)$ the periodicity of $g(z ; \beta ; \Delta)$ in $\Delta$ leads to the same periodicity of $\zeta$ in $\Delta$ :

$$
\zeta(\Delta+1)=\zeta(\Delta)
$$

and also to the relation: $\zeta(-\Delta)=\zeta(\Delta)$ *.

There we use also the fact that the rectangle $\mathscr{R}_{0, L}$ is invariant under complex conjugation. This means for $\zeta=\xi+i \eta$ :

$$
\xi(\Delta)=\xi(-\Delta) \text { and } \eta(-\Delta)=-\eta(\Delta) \text {. }
$$

Lemma 3. The function $\zeta=\zeta(\Delta)$ fulfills the following relations:

(a) $\zeta(0)=0, \zeta(1 / 2)=x_{0}(\beta)$,

(b) $\zeta^{\prime}(0)=-i 2 \pi /(\beta+1), \zeta^{\prime}(1 / 2)=2 \pi e^{-\beta x_{0}(\beta)} /\left(e^{-x_{0}(\beta)}-\beta e^{-\beta x_{0}(\beta)}\right)$

$\xi^{\prime}(0)=-8 \pi^{2} /(\beta+1)^{3}$,

(c) $\xi^{\prime}(\Delta)<0$ for $0<\Delta \bmod 1<1 / 2, \eta^{\prime}(\Delta)<0$ for $0 \leq \Delta \bmod 1<\tilde{\Delta}$ for some $0<\tilde{\Delta}<1 / 2$,

For $\Delta \rightarrow 0$ we have

(d) $\xi(\Delta)=-8 \pi^{2} /(\beta+1)^{3} \Delta^{2}+0\left(\Delta^{4}\right), \eta(\Delta)=-2 \pi /(\beta+1) \Delta+0\left(\Delta^{3}\right)$.

Proof. Because $g(0 ; \beta ; 0)=0$ and $0 \in \mathscr{R}_{0, L}$ we have from Lemma 1 that $\zeta(0)=0$. In complete analogy one argues for $\zeta\left(\frac{1}{2}\right)=x_{0}(\beta)$. Let us next consider the different properties of the function $\zeta^{\prime}(\Delta)$. From $g(z ; \beta ; \Delta)=0$ we get the two equations for $z=x+i y$ :

$$
e^{-x} \cos y+e^{-\beta x} \cos (\beta y+2 \pi \Delta)=2
$$




$$
e^{-x} \sin y+e^{-\beta x} \sin (\beta y+2 \pi \Delta)=0 .
$$

From (11) it is clear that $\sin y=0$ if and only if $\sin (\beta y+2 \pi \Delta)=0$. For $z \in \mathscr{R}_{0, L}$ this can happen only for $y=\eta(\Delta)=0$ and $\Delta=\frac{1}{2} n, n \in \mathbb{Z}$. But then $\sin \eta(\Delta) \neq 0, \sin (\beta \eta(\Delta)+2 \pi \Delta) \neq 0$ for $\Delta \neq n / 2, n \in \mathbb{Z}$, especially $\operatorname{sign} \sin \eta(\Delta)=-\operatorname{sign} \sin (\beta \eta(\Delta)+2 \pi \Delta)$ for $0<\Delta \bmod 1<\frac{1}{2}$. For these values we can also write the two equations (10), (11) as follows

$$
\begin{gathered}
e^{(1-\beta) \xi(\Delta)}=-\sin \eta(\Delta) / \sin (\beta \eta(\Delta)+2 \pi \Delta) \\
e^{\xi(\Delta)}=1 / 2 \sin ((\beta-1) \eta(\Delta)+2 \pi \Delta) / \sin (\beta \eta(\Delta)+2 \pi \Delta) .
\end{gathered}
$$

The second equation shows that sign $\sin ((\beta-1) \eta(\Delta)+2 \pi \Delta)=\operatorname{sign} \sin$ $(\beta \eta(\Delta)+2 \pi \Delta)=-\operatorname{sign} \sin \eta(\Delta)$ for $\Delta \neq \frac{n}{2}, n \in \mathbb{Z}$.

Let us calculate next $\zeta^{\prime}(\Delta)$ with the help of relation (9) and

$$
\frac{\partial g(z ; \beta ; \Delta)}{\partial \Delta}=-2 \pi i \exp (-\beta z-2 \pi i \Delta):
$$

After some trivial algebra we find

$$
\begin{aligned}
& \xi^{\prime}(\Delta)=-2 \pi \sin ((\beta-1) y+2 \pi \Delta) e^{-(\beta+1) x} /\left.\left|\frac{\partial g(z ; \beta ; \Delta)}{\partial z}\right|^{2}\right|_{z=x+i y=\zeta(\Delta)} \\
& \eta^{\prime}(\Delta)=-2 \pi\left(\beta e^{-2 \beta x}+e^{-(\beta+1) x} \cos ((\beta-1) y+2 \pi \Delta)\right) /\left.\left|\frac{\partial g(z ; \beta ; \Delta)}{\partial z}\right|^{2}\right|_{z=\zeta(\Delta)}
\end{aligned}
$$

Inserting the values $\zeta(0)=0$ and $\zeta\left(\frac{1}{2}\right)=x_{0}(\beta)$ then gives:

$$
\begin{aligned}
& \xi^{\prime}(0)=0, \eta^{\prime}(0)=-2 \pi /(\beta+1) \text { and therefore } \zeta^{\prime}(0)=-i 2 \pi /(\beta+1) \\
& \xi^{\prime}(1 / 2)=0, \eta^{\prime}(1 / 2)=2 \pi \exp \left(-\beta x_{0}(\beta)\right) /\left(\exp -x_{0}(\beta)-\beta \exp -\beta x_{0}(\beta)\right)
\end{aligned}
$$

which leads to

$$
\zeta^{\prime}(1 / 2)=i 2 \pi \exp -\beta x_{0}(\beta) /\left(\exp -x_{0}(\beta)-\beta \exp -\beta x_{0}(\beta)\right) .
$$

For $\Delta \neq \frac{n}{2}$ we know that $\sin ((\beta-1) \eta(\Delta)+2 \pi \Delta) \neq 0$ and has opposite sign to $\sin (\eta(\Delta))$. But $\eta^{\prime}(0)<0$ and $\eta(0)=0$ show therefore sin $\eta(\Delta)<0$ for $0<\Delta<\frac{1}{2}$ respectively $0<\Delta \bmod 1<\frac{1}{2}$.

Relation (14) then shows that $\xi^{\prime}(\Delta)<0$ for these $\Delta$ values. Expression (15) for $\eta^{\prime}(\Delta)$ can be rewritten with the help of relations (12) 
and (13) as follows

$$
\eta^{\prime}(\Delta)=-2 \pi /\left.\left(\left|\frac{\partial g(z ; \beta ; \Delta)}{\partial z}\right|\right)^{2} e^{-2 \beta x}\left(\beta-1+2 e^{\beta x} \cos (\beta y+2 \pi \Delta)\right)\right|_{z=\zeta(\Delta)} .
$$

Because $\eta^{\prime}(0)<0$ and $\eta^{\prime}\left(\frac{1}{2}\right)>0$ there exists a $0<\tilde{\Delta}<\frac{1}{2}$ such that $\eta^{\prime}(\tilde{\Delta})=0$. This $\tilde{\Delta}$ is the solution of the equation

$$
\beta-1+2 e^{\beta \zeta(\tilde{\Delta})} \cos (\beta \eta(\tilde{\Delta})+2 \pi \tilde{\Delta})=0 .
$$

The asymptotic formulas for $\Delta \rightarrow 0$ then follow immediately from a, b, and $c$, together with real analyticity of $\zeta(\Delta)$.

This concludes the proof of Lemma 3. Theorem II then follows from Lemma 1 to Lemma 3.

Let us conclude with some remarks: How these results can be used to improve the results on the long time behaviour of correlation functions for certain turbulent systems mentioned in the introduction will be discussed in [13]. Another problem which arises immediately from our result concerns the zero's of more general exponential sums of the form $e^{-z}+e^{-\beta_{1} z}++e^{-\beta_{n} z}-(n+1)$. We expect that more or less analogous results hold and that the location of the zero's is closely related to the simultaneous Diophantine distances $\Delta_{m}\left(\beta_{1}\right), \ldots$, $\Delta_{m}\left(\beta_{n}\right)$ respectively the best simultaneous Diophantine approximation denomintors $q_{n}$ of the numbers $\beta_{1}, \ldots, \beta_{n}[14]$.

\section{Literature}

[1] Ruelle, D., Flots qui ne mélangent pas exponentiellement, C. R. Acad. Sci. Paris, 296 (1983), 191-193.

[2] Pollicott, M., On the rate of mixing of Axiom A flows. Preprint I. H. E. S. (1985), Invent. math. 81 (1985) 413-426.

[3] Parry, W., An analogue of the prime number theorem for shifts of finite type and their suspensions, Israel J. Math., 45 (1983), 41-52.

[4] Pollicott, M., Meromorphic extensions of generalized zeta functions, Preprint Math. Institute of Warwick (1984).

[5] Bohr, H., Zur Theorie der fastperiodischen Funktionen III, Acta math., 47 (1926), $237-281$.

[6] Jessen, B., Tornehave, H., Mean motions and zeros of almost periodic functions, Acta math., 77 (1945), 137-279.

[7] Tamarkin, J., The zeros of certain integral functions, J. Lond. Math. Soc., 2 (1927), 66-69.

[ 8 ] Polya, G., Geometrisches über die Verteilung der Nullstellen spezieller ganzer transzendenter Funktionen, S. -B. Bayer. Akad. Wiss. Munchen, (1920), 285-290. 
[9] Ritt, J., On the zeros of exponential polynomials, Trans. Amer. Math. Soc., 31 (1929), 680-686.

[10] Lang, S., "Introduction to Diophantine Approximations', p. 10, Addison-Wesley, Reading, Mass. 1966.

[11] Alexandroff, P., Hopf, H., "Topologie", Bd. 1, p. 459, Chelsea Publ. Comp., Bronx, N. Y. 1972.

[12] ibid, p. 472.

[13] Lohöfer, G., Ph-D. Thesis, RWTH Aachen (1985).

[14] Lagarias J., Some new results in simultaneous Diophantine Approximation. In "Proc. of the Queen Univ. Number Theory Conf. 1979" ed. P. Ribenboim, Queens Papers in Pure and Appl. Math., 54 (1980), 453-474.

[15] Mayer, D., Roepstorff, G., Strange attractors and asymptotic measures of discrete time dissipative systems, J. Stat. Phys., 31 (1983), 309-326. 
